\title{
Cannabis/Cannabinoids for Treating COVID-19 Associated Neuropsychiatric Complications
}

\author{
Jag H. Khalsa ${ }^{1,2,3}$. Sanjay B. Maggirwar ${ }^{3}$. Greg Bunt ${ }^{4}$
}

Received: 4 May 2021 / Accepted: 19 August 2021 / Published online: 16 October 2021

(c) The Author(s), under exclusive licence to Springer Science+Business Media, LLC, part of Springer Nature 2021

\begin{abstract}
COVID-19 epidemic has resulted in devastating mortality and morbidity consisting of socioeconomic and health effects that have included respiratory/pulmonary, cardiovascular, mental health and neurological consequences such as anxiety, depression, and substance use. Several effective vaccines have been developed and extensive efforts are underway to develop therapeutics to treat COVID-19. Cannabis and/or its product-cannabidiol (CBD) are being advertised for the treatment of COVID-19 associated mental/neurological complications and substance use disorders. However, research reviewed shows that there is insufficient data from clinical studies to support the use of cannabis or CBD for the treatment of COVID-19 associated mental health and neurological complications. Additional basic and clinical research is suggested to develop cannabis or cannabidiol for the treatment of mental health problems associated with coronavirus infection and or substance use disorders. In the meantime, it is important that the addiction physician/psychiatrist must caution while prescribing or recommending cannabis or CBD for treating such clinical indications.
\end{abstract}

Keywords COVID-19 $\cdot$ Cannabis $\cdot$ Cannabidiol $\cdot$ CBD $\cdot$ Neuropsychiatric

In 1918, the Spanish flu killed millions of people worldwide. Once again, the world is facing a devastating viral pandemic where almost 189 million people have been infected with a novel corona virus, known as severe acute respiratory syndrome cornonavirus-2 (SARS-Cov2), that binds to a host cell through its target angiotensin-converting enzyme 2 (ACE 2 ) receptor (Cevik et al. 2020), leading to corona virus induced disease,

Jag H. Khalsa

jkhalsa@yahoo.com; jag.khalsa@nih.gov

Sanjay B. Maggirwar

smaggirwar@gwu.edu

Greg Bunt

buntmd@aol.com

1 Medical Consequences of Drug Abuse and Infections Branch, National Institute on Drug Abuse, NIH, Bethesda, MD, USA

24924 McNair Place, Aldie, VA 20105, USA

3 Department of Microbiology, Immunology and Tropical Medicine, The George Washington University School of Medicine and Health Sciences, 2300 I Street, NW, Ross Hall Room 502A, Washington, DC 20037, USA

4 American Board of Psychiatry and Neurology, NYU Medical School, 550 First Ave, New York, NY 10016, USA known as COVID-19, that has resulted in more than 4 million deaths worldwide (Coronavirus Resource Center, 2021). The facts and not the 'alternate facts', are clear, that the COVID-19 pandemic has resulted in a wide range of unprecedented economic, social, and short-and long-term medical/health consequences including severe acute respiratory syndrome (SARS) (DeFelice et al. 2020), hypertension, myocardial injury (Clerkin et al. 2020), anxiety, depression, tremors, seizures, and impaired consciousness (Whittaker et al. 2020; Acharya et al. 2020; Varatharaj et al. 2020). It is estimated that about $45 \%$ of adults in the US suffered from impaired mental health because of worry and stress over the virus (Krizinger et al. 2020). According to CDC, people with pre-existing health conditions including asthma, coronary heart disease, hypertension, diabetes, immunocompromised state, and dementia also might be at increased risk from COVID-19 (CDC 2020). Furthermore, the conditions such as imposed shelter-in-place, physical distancing, low esteem, social isolation, and potential financial distress from job loss might further lead to mental health problems, increased substance use, and possibly suicides. Finally, limited access to mental health care and addiction treatment for treating substance use disorders (SUDs) may also in part exacerbate COVID-19 related complications, making it necessary to use telemedicine to treat people with SUDs. 
Several vaccines are being successively used to prevent further Covid-19-related morbidity and mortality. Besides using currently available therapeutics like remdesivir, dexamethasone, and convalescent plasma, extensive efforts worldwide are underway to develop new therapeutics to combat the coronavirus induced symptomatic disease (COVID-19) and associated complications. Some of the newer therapeutics under development also include SNG001, an inhalable beta interferon delivered by nebulizer, (Synairgen); AZD7442, a long-acting monoclonal antibody combination as both an infusion and an intramuscular injection (AstraZeneca); and Camostat mesilate, an orally administered serine protease inhibitor that may block SARS-CoV-2 virus from entering cells (Uno 2020; Sagent Pharmaceuticals), under the NIH's program of Accelerating COVID-19 Therapeutic Interventions and Vaccines (ACTIV). [(COVID-19 Therapeutics Prioritized for Testing in Clinical Trials I National Institutes of Health (NIH)), accessed 03282021]. In addition, the second set of facts are that the internet is filled with suggestions that cannabis or one of its many cannabinoids including cannabidiol (CBD) could be used for the treatment of not only coronavirus infection induced inflammation via cytokine storm (Hu et al. 2021), but also anxiety, depression, post-traumatic disorder (PTSD), and cognitive decline seen in patients with COVID19. Because of the recent changes in legalization of cannabis or CBD in most US states, cannabis and/or CBD products have become easily accessible as over the counter products for treating a wide variety of clinical conditions. Therefore, in this short review, we will show that the currently available research from published clinical studies and trials is insufficient to support the use of cannabis or cannabinoids including CBD for the treatment of mental health problems in people with or without coronavirus infection or SUDs and more basic and clinical research is needed to support such claims.

The use of cannabis as medicine is not new. In year 2737 BC, the Chinese Emperor Shen-Nung was the first to describe the medicinal value of cannabis ( $\mathrm{Li} 1974)$ and since then many preparations of cannabis have been used for recreational and medicinal purposes (Gaoni and Mechoulam 1971; Mechoulam and Gaoni 1967; Breivogel et al. 1998). Cannabis sativa, Linn. is a complex plant that has 560 identified and characterized chemicals (ElSohly et al. 2017), of which 104 are classified as cannabinoids, while the rest are terpenes and flavinoids. Cannabinoids like cannabichromene (CBC), cannabidolic acid (CBDA), cannabidiol (CBD), delta-9-tetrahydrocannabivarin $\left(\Delta^{9}\right.$-THCV), cannabinol (CBN), delta-9-tetrahyrocannabinol ( $\Delta$-THC), cannabinol (CBL), delta-9-tetrahydrocannabivaric acid $\left(\Delta^{9}\right.$-THCAA), are the major components of $C$. sativa, while cannabigerolic acid (CBGA), cannabigerol (CBG) and delta-8-tetrahydrocannabinol $(\triangle 8$-HC) are minor constituents (Gul et al. 2018) and have been postulated to have some medicinal value (Smith 1998). Curre ${ }^{9}$ ntly, data on safety, pharmacology, and clinical efficacy on any of these cannabinoids is insufficient to support their use as medicine (Khalsa et al. 2019). Only two cannabinoidsthe psychoactive delta-9-tetrahydrocannabinol (THC) and non-psychoactive cannabidiol (CBD) - have been extensively studied for their potential therapeutic applications. Although CBD has potential to treat a wide range of clinical conditions like disorders of generalized anxiety, social anxiety, panic, and PTSD (Blessing et al. 2015), and depressive disorders via serotonergic pathways (de Mello et al. 2014) and endocannabinoid system (Ashton and Moore 2011), more clinical research from well-designed clinical trials is needed to support its use in treating anxiety and depressive disorders and bipolar disorders (Ashton et al. 2005). CBD also may be effective in promoting wakefulness, via triggering increased dopamine levels in either lateral hypothalamus or dorsal raphe nuclei, the areas of brain responsible for wakefulness, suggesting that CBD could treat sleeping disorders such as narcolepsy (MurilloRodriguez et al. 2008). In fact, CBD did improve the quality and quantity of sleep of a 10-year-old young patient with PTSD, likely due to its anxiety-relieving benefits (Shannon and Opila-Lehman 2016). But this is in one case report. There is a clear paucity of data from well-designed clinical trials to support the use of cannabis, CBD, or any other cannabinoid for treating sleep disorders, either anxiety, depression, other neurological complications associated with COVID-19 or patients with SUDs. However, there is a silver lining-the fact that CBD inhibits SARSCov-2 replication and promotes the host innate response in humans (Nguyen et al. 2021), a clear positive aspect of CBD, and as evidenced by data from the NIH's database (http://clinicaltrials.gov; accessed July 13, 2021), several investigators are studying either the dose tolerability or the efficacy of CBD in treating anxiety or depression. However, it remains to be seen when clinical data would be available from these clinical studies that would support the use of CBD for treating any of the mental or neuropsychiatric complications. In the meantime, it must be pointed out that currently, there are no data from any clinical studies or trials that have been considered adequate by a regulatory body like the US FDA to support the use of CBD for the treatment of various mental or neuropsychiatric conditions as being promoted.

In the case of cannabis, cannabinoids including CBD for treating substance use disorders, limited research does suggest that CBD could potentially treat patients with opiate, cannabis, and tobacco use disorders (OUD, CUD, TUD) (Chye et al. 2019). Earlier studies showed that legalization of medical marijuana reduced the number of over-dose deaths from opioid pain relievers (Finney et al. 2015), and 
that medical marijuana laws significantly reduced prescribing of opioids for pain (Bradford and Bradford 2016). But more recently, Hurd et al. (2019) reported that acute administration of CBD to heroin abstinent patients with OUD significantly reduced craving, anxiety, heart rate and salivary cortisol without causing adverse effects. In the case of cannabis use disorder (CUD), CBD reduced euphoria, fewer depressive and psychotic-like symptoms and improvements in attentional improvements, verbal learning, and memory without impairing cognition when smoking cannabis, suggesting that prolonged therapy with CBD may be a useful adjunct therapy for treating cannabis dependence (Slowij et al. 2018; Weise and Wilson-Poe 2018). Trigo et al. (2018) also reported that nabiximols (CBD + THC in 1:1 ratio [Sativex®]) combined with Motivational Enhancement Therapy and Cognitive Behavioral Therapy (MET/CBT), reduced the cannabis use and craving but not withdrawal symptoms in chronic cannabis users. Incidentally, CBD is approved for treating a rare form of epilepsy-LennoxGastaut and Dravet syndromes in young children, another positive effect of CBD as medicine. Regarding tobacco use disorder, in a study of 24 tobacco smokers, CBD inhaler reduced the number of tobacco cigarettes by $40 \%$ when compared to placebo (Morgan et al. 2013); and a single dose of $800 \mathrm{mg}$ oral dose of CBD reduced the salience and pleasantness of cigarette cues but did not influence tobacco craving or withdrawal or any subjectively rated side effects (Hindocha et al. 2018). These studies in a small number of patients provide us a positive signal that CBD has a great potential to treat a wide range of clinical conditions, but not without conducting additional well-designed clinical trials to support its use as therapy for mental conditions including SUDs. It is also important to keep in mind that cannabinoids may result in adverse pharmacokinetic/pharmacodynamic drugdrug interactions with other pharmacologic agents used in the treatment of patients with COVID-19 (Land et al. 2020) or patients with substance use disorders.

In clinical practice, $\mathrm{CBD}$ is available as: (a) pharmaceutical grade CBD that may be prescribed off-label; (b) as over the counter CBD products in pharmacies; (c) as CBD products obtained through internet sources; and (d) CBD preparations from cannabis dispensaries. Efficacy, safety, and tolerability data at widely varying doses however are needed for the physician to make informed clinical recommendations and decisions. Recent survey data of addiction physicians' views from an ASAM symposium indicated that most addiction physicians have a considerable interest in the utilization of CBD, both pharmaceutical grade and non-pharmaceutical grade, in clinical practice particularly for its potential for the treatment of pain; however, substantially more research is needed for physicians to recommend or prescribe $\mathrm{CBD}$ as a medication for a range of clinical disorders. Therefore, it is imperative that the clinicians treating patients with any of the above COVID19 associated mental or neurological conditions and substance use disorders inform their patients about the lack of sufficient clinical evidence for the use of CBD or cannabis and discourage them from using non-FDA approved cannabis, cannabinoids including CBD or THC for selftreating themselves for COVID-19 related health problems including SUDs.

In summary, research reviewed clearly suggests that, although CBD, that is approved for treating epilepsy in young children, and in combination with THC as Sativex, for treating symptoms of multiple sclerosis, and has potential to treat a wide range of clinical indications, there is insufficient clinical evidence to support the use of cannabis or its highly advertised productCBD in the treatment of COVID-19-asociated mental health or neuropsychiatric complications at this time. More systematic evaluation of cannabis or CBD in large clinical trials is essential prior to endorsing wider use of CBD or other cannabis products for alleviation of mental health symptomatology. Therefore, we suggest that as per the FDA's GLPs (good laboratory practice and good clinical practice guidelines), well-designed basic, clinical studies, and randomized double-blind, placebo-controlled clinical trials, including studies of drug-drug interactions, where appropriate, be conducted to access whether cannabis, or individual cannabinoids such as THC, CBD, alone or in combination are safe and effective in treating mental disorders including cognitive decline, anxiety, depression, and/or PTSD seen in patients with COVID-19 or people with SUDs. Furthermore, and more importantly, because of variability of chemical constituents in cannabis from different sources, cannabis used in research must be of research grade and well characterized in terms of its chemical composition as per the FDA's good manufacturing guidelines (GMPs).

Acknowledgements Dr. Jag H. Khalsa is grateful to the US National Institute on Drug Abuse, a component of the National Institutes of Health, Department of Health and Human Services, for an opportunity to serve as a Special Volunteer following his retirement on October 31, 2017, after 30+ years as the Chief, Medical Consequences of Drug Abuse and Infections Branch.

Author Contributions Conceived and drafted by JK, then all authors contributed equally to the manuscript.

Funding JK: Self; GB: Samaritan/Day Top Village; SBM: R01NS066801; R01AG 054325.

\section{Declarations}

Conflicts of interest None for JK, GB, SBM. 


\section{References}

Acharya A, Kevadiya BD, Gendelman HE et al (2020) SARS-Cov-2 infection leads to neurological dysfunction. Neuroimmune Pharmacol 15(2):167-173

Ashton CH, Moore PB (2011) Endocannabinoid system dysfunction in mood and related disorders. Acta Psychiatric Scand 124(4):250-261

Ashton CH, Moore PB, Gallagher P, Young AH (2005) Cannabinoids in bipolar affective disorder: a review and discussion of their therapeutic potential. J Psychopharmacol (3): 293-300. https:// doi.org/10.1177/0269881105051541. PMID:15888515.

Blessing EM, Steenkamp MM, Manzanares J et al (2015) Cannabidiol as a potential treatment for anxiety disorders. Neurotherapeutics $12: 825-836$

Bradford AC, Bradford WD (2016) Medical marijuana laws reduce prescription medication use in medicare part D. Health Aff Proj Hope 35(7):1230-1236

Breivogel CS, Selley DE, Childers SR (1998) Cannabinoid receptor agonist efficacy for stimulating [35S]GTPgammaS binding to rat cerebellar membranes correlates with agonist-induced decreases in GDP affinity. J Biol Chem 273:16865-16873

Centers for Disease Control and Prevention, Coronavirus disease 2019 (COVID-19), People with certain medical conditions, CDC, 2020. https://www.cdc.gov/coronavirus/2019-ncov/need-extra-precautions/ people-with-medical-conditions.html. Accessed 07 March 2020

Cevik M, Kuppalli K, Kindrachuk J, Peiris M (2020) Virology, transmission, and pathogenesis of SARS-CoV-2. BMJ 23(371):m3862. https://doi.org/10.1136/bmj.m3862

Chye Y, Christensen E, Solowij N et al (2019) The endocannabinoid system and cannabidiol's promise for the treatment of substance use disorder. Front Psychiatry 10:63. https://doi.org/10.3389/ fpsyt.2019.00063

Clerkin KJ, Fried JA, Raikhelkar J et al (2020) Covid-19 and cardiovascular disease. Circulation 141:1648-1655

Coronavirus Resource Center, Coronavirus Case Tracker, Johns Hopkins University, Baltimore, Maryland. 2021. https://coronavirus.jhu. edu/. Accessed 07 May 2021

De Felice FG, Tovar-Moll F, Moll J et al (2020) Severe acute respiratory syndrome coronavirus 2 (SARS-CoV-2) and the central nervous system. Trends Neurosci 43(6):355-357

de Mello Schier AR, de Oliveira Ribeiro NP, Coutinho DS et al (2014) Antidepressant-like and anxiolytic-like effects of cannabidiol: a chemical compound of Cannabis sativa. CNS Neurol Disord Drug Targets 13:953-960

ElSohly MA, Radwan MM, Gul W, Chandra S, Galal A (2017) Phytochemistry of Cannabis sativa L. Prog Chem Org Nat Prod 103:1-36

Finney JW, Humphreys K, Harris AHS (2015) What ecologic analyses cannot tell us about medical marijuana legalization and opioid pain medication mortality. JAMA Intern Med 175(4):655-656

Gaoni Y, Mechoulam R (1971) The isolation and structure of delta1-tetrahydrocannabinol and other neutral cannabinoids from hashish. J Am Chem Soc 93:217-224

Gul W, Gul SW, Chandra S, Lata H, Ibrahim EA, ElSohly MA (2018) Detection and quantification of cannabinoids in extracts of cannabis sativa roots using LC-MS/MS. Planta Med 84:267-271

Hindocha C, Freeman TP, Grabski M et al (2018) Cannabidiol reverses attentional bias to cigarette cues in a human experimental model of tobacco withdrawal. Addiction 113:1696-1705

Hu B, Huang S, Yin L (2021) The cytokine storm and COVID-19. J Med Virol 93(1):250-256. https://doi.org/10.1002/jmv.26232
Hurd YL, Spriggs S, Alishayev J et al (2019) Cannabidiol for the reduction of cue-induced craving and anxiety in drug-abstinent individuals with heroin use disorder: a double-blind randomized placebo-controlled trial. Am J Psychiatry 176(11):911-922

Khalsa J, Bunt G, Galanter M, Wetterau N (2019) Cannabidiol as medicine: are we there? Int Addict Rev 2(2):5-18

Krizinger A, Kearney A, Hamel L, Brodie M (2020) KFF Health Tracking Poll-Early April 2020: the impact of coronavirus on life in America. https://www.kff.org/coronavirus-covid-19/report/kffhealth-tracking-poll-early-april-2020/. Accessed 07 May 2020

Land MH, MacNair L, Thomas BF, Peters EN, Bonn-Miller MO (2020) Letter to the editor: possible drug-drug interactions between cannabinoids and candidate COVID-19 drugs. Cannabis Cannabinoid Res 5:340. https://doi.org/10.1089/can.2020.0054

Li HL (1974) An archaeological and botanical account of cannabis in China. Econ Bot 28:437-448

Mechoulam R, Gaoni Y (1967) Recent advances in the chemistry of hashish. Fortschr Chem Org Naturst 25:175-213

Morgan CJ, Das RK, Joye A et al (2013) Cannabidiol reduces cigarette consumption in tobacco smokers: preliminary findings. Addict Behav 38:2433-2436

Murillo-Rodriguez E, Millan-Aldaco D, Palomero-Rivero M, Mechoulam $\mathrm{R}$ et al (2008) The nonpsychoactive Cannabis constituent cannabidiol is a wake-inducing agent. Behav Neurosci 122:1378-1382

Nguyen LC, Yang D, Nicolaescu V et al (2021) Cannabidiol inhibits SARS-CoV-2 replication and promotes the host innate immune response. Preprint. bioRxiv. https://doi.org/10.1101/2021.03.10. 432967

Shannon S, Opila-Lehman J (2016) Effectiveness of cannabidiol oil for pediatric anxiety and insomnia as part of posttraumatic stress disorder: a case report. Perm J 20:108-111

Smith DE (1998) Review of the American Medical Association Council on Scientific Affairs report on medical marijuana. J Psychoactive Drugs 30:127-136

Solowij N, Broyd SJ, Beale C et al (2018) Therapeutic effects of prolonged cannabidiol treatment on psychological symptoms and cognitive function in regular cannabis users: a pragmatic openlabel clinical trial. Cannabis Cannabinoid Res 3:21-34

Trigo JM, Soliman A, Quilty LC et al (2018) Nabiximols combined with motivational enhancement/cognitive behavioral therapy for the treatment of cannabis dependence: a pilot randomized clinical trial. PLoS ONE 13:e0190768

Uno Y (2020) Camostat mesilate therapy for COVID-19. Intern Emerg Med 15(8):1577-1578. https://doi.org/10.1007/ s11739-020-02345-9

Varatharaj A, Thomas N, Ellul MA, et al. on behalf of the CoroNerve Study Group (2020) Neurological and neuropsychiatric complications of COVID-19 in 153 patients: a UK-wide surveillance study. Lancet Psychiatry. https://doi.org/10.1016/S2215-0366(20) 30287-X

Weise B, Wilson-Poe AR (2018) Emerging evidence for cannabis' role in opioid use disorder. Cannabis Cannabinoid Res 3(1):179-189

Whittaker A, Anson M, Harky A et al (2020) Neurological manifestations of COVID19: a systematic review and current update. Acta Neurol Scand 142(1):14-22

Publisher's Note Springer Nature remains neutral with regard to jurisdictional claims in published maps and institutional affiliations.

Disclaimer The statements in this paper are of the authors' only and do not reflect the official position of any of their organizations. 